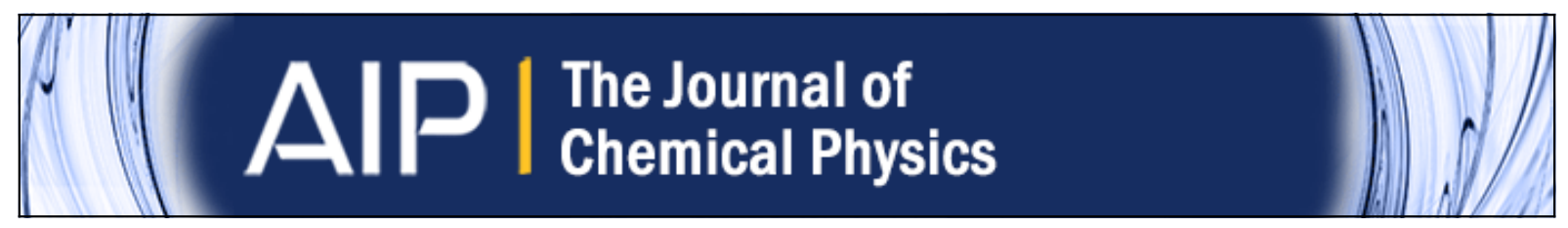

\title{
Spatial distribution of intra-molecular water and polymeric components in polyelectrolyte dendrimers revealed by small angle scattering investigations
}

Bin Wu, Xin Li, Changwoo Do, Tae-Hwan Kim, Chwen-Yang Shew, Yun Liu, Jun Yang, Kunlun Hong, Lionel Porcar, Chun-Yu Chen, Emily L. Liu, Gregory S. Smith, Kenneth W. Herwig, and Wei-Ren Chen

Citation: The Journal of Chemical Physics 135, 144903 (2011); doi: 10.1063/1.3651364

View online: http://dx.doi.org/10.1063/1.3651364

View Table of Contents: http://scitation.aip.org/content/aip/journal/jcp/135/14?ver=pdfcov

Published by the AIP Publishing

\section{Articles you may be interested in}

Molecular dynamics and neutron scattering study of the dependence of polyelectrolyte dendrimer conformation on counterion behavior

J. Chem. Phys. 137, 064902 (2012); 10.1063/1.4742190

Conformational effect on small angle neutron scattering behavior of interacting polyelectrolyte solutions: A perspective of integral equation theory

J. Chem. Phys. 137, 024907 (2012); 10.1063/1.4732516

Structured water in polyelectrolyte dendrimers: Understanding small angle neutron scattering results through atomistic simulation

J. Chem. Phys. 136, 144901 (2012); 10.1063/1.3697479

Probing the extent of the $\mathrm{Sr} 2+$ ion condensation to anionic polyacrylate coils: A quantitative anomalous smallangle $x$-ray scattering study

J. Chem. Phys. 127, 154908 (2007); 10.1063/1.2787008

Influence of counterion valency on the scattering properties of highly charged polyelectrolyte solutions J. Chem. Phys. 114, 3299 (2001); 10.1063/1.1336148

How can you REACH $100 \%$

of researchers at the Top 100

Physical Sciences Universities?

With The Journal of Chemical Physics.

AIP $\left.\right|_{\text {Chemical Physics }} ^{\text {The Journal of }}$

THERE'S POWER IN NUMBERS. Reach the world with AIP Publishing.

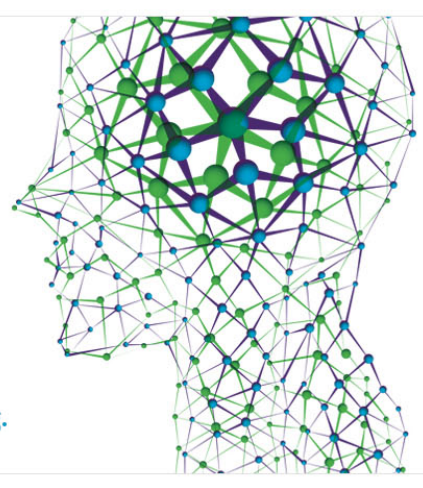




\title{
Spatial distribution of intra-molecular water and polymeric components in polyelectrolyte dendrimers revealed by small angle scattering investigations
}

\author{
Bin $\mathrm{Wu},{ }^{1,2}$ Xin $\mathrm{Li}^{3,}{ }^{3, a)}$ Changwoo Do, ${ }^{1,4}$ Tae-Hwan Kim, ${ }^{5}$ Chwen-Yang Shew, ${ }^{6}$ Yun Liu, ${ }^{7,8}$ \\ Jun Yang, ${ }^{9}$ Kunlun Hong, ${ }^{9, a)}$ Lionel Porcar, ${ }^{10}$ Chun-Yu Chen, ${ }^{11}$ Emily L. Liu, ${ }^{2}$ \\ Gregory S. Smith, ${ }^{1}$ Kenneth W. Herwig, ${ }^{12}$ and Wei-Ren Chen ${ }^{1,13,14, a)}$ \\ ${ }^{1}$ Neutron Scattering Science Division, Oak Ridge National Laboratory, Oak Ridge, Tennessee 37831, USA \\ ${ }^{2}$ Department of Mechanical, Aerospace \& Nuclear Engineering, Rensselaer Polytechnic Institute, Troy, \\ New York 12180, USA \\ ${ }^{3}$ Department of Physics, Indiana University, Bloomington, Indiana 47405-7105, USA \\ ${ }^{4}$ Jülich Centre for Neutron Science, Forschungszentrum Jülich, 52425 Jülich, Germany \\ ${ }^{5}$ Neutron Science Division, Korea Atomic Energy and Research Institute, Daejon 305-600, South Korea \\ ${ }^{6}$ City University of New York, College of Staten Island, Staten Island, New York 10314, USA \\ ${ }^{7}$ The NIST Center for Neutron Research, National Institute of Standards and Technology, Gaithersburg, \\ Maryland 20899-6100, USA \\ ${ }^{8}$ Department of Chemical Engineering, University of Delaware, Newark, Delaware 19716, USA \\ ${ }^{9}$ Center for Nanophase Materials Sciences, Oak Ridge National Laboratory, Oak Ridge, Tennessee 37831, USA \\ ${ }^{10}$ Institut Laue-Langevin, B.P. 156, F-38042 Grenoble CEDEX 9, France \\ ${ }^{11}$ National Synchrotron Radiation Research Center, Hsinchu 30076, Taiwan \\ ${ }^{12}$ Neutron Facilities Development Division, Oak Ridge National Laboratory, Oak Ridge, Tennessee 37831, USA \\ ${ }^{13}$ Department of Chemical and Biomolecular Engineering, The University of Tennessee, Knoxville, \\ Tennessee 37996-2200, USA \\ ${ }^{14}$ Joint Institute for Neutron Sciences, Oak Ridge National Laboratory, Oak Ridge, Tennessee 37831, USA
}

(Received 22 June 2011; accepted 23 September 2011; published online 14 October 2011)

\begin{abstract}
An experimental scheme using contrast variation small angle neutron scattering technique is developed to investigate the structural characteristics of amine-terminated poly(amidoamine) dendrimers solutions. Using this methodology, we present the dependence of both the intra-dendrimer water and the polymer distribution on molecular protonation, which can be precisely adjusted by tuning the $p \mathrm{H}$ of the solution. Assuming spherical symmetry of the spatial arrangement of the constituent components of dendrimer, and that the atomic ratio of hydrogen-to-deuterium for the solvent residing within the cavities of dendrimer is identical to that for the solvent outside the dendrimer, the intradendrimer water distribution along the radial direction is determined. Our result clearly reveals an outward relocation of the peripheral groups, as well as enhanced intra-dendrimer hydration, upon increasing the molecular protonation and, therefore, allows the determination of segmental backfolding in a quantitative manner. The connection between these charge-induced structural changes and our recently observed progressively active segmental dynamics is also discussed. @ 2011 American Institute of Physics. [doi:10.1063/1.3651364]
\end{abstract}

\section{INTRODUCTION}

Highly branched dendritic macromolecules, commonly referred to as dendrimers, form a unique category of synthetic polymers. ${ }^{1,2}$ They have technological interest since they find application in a wide variety of contexts. ${ }^{1,3,4}$ In the scientific community they have also received considerable attention, especially due to the structural duality arising from their well-defined particle-like resemblance and flexible, porous polymeric architecture, presenting a hybrid between compact colloids and linear polymeric chains. ${ }^{1,2}$ Among this class of synthetic macromolecules, polyamidoamine dendrimers (PAMAM), with ethylenediamine cores and polyamidoamino units with repeating dendritic branching, have been one of the most studied systems not only from the aforementioned scientific viewpoint, but also in terms of their many potential

\footnotetext{
a) Authors to whom correspondence should be addressed. Electronic addresses: chenw@ornl.gov, hongkq@ornl.gov, and li300@indiana.edu.
}

applications as delivery agents of guest materials in a wide variety of contexts, including drug, DNA, and metal ions.,

When dissolved in aqueous solutions, both the terminal primary amines and tertiary amines located at the branching points in the interior of PAMAM dendrimers can be charged via protonation by acidifying the solution, and therefore, the dendrimer possesses the properties of a polyelectrolyte. ${ }^{1}$ In practical applications, such as guest transport vehicles, polyelectrolyte dendrimers are designed to operate in different aqueous environments with varying conditions which could cause change of their charge conditions. ${ }^{1,3,4}$ To facilitate their use in these applications, a clear understanding of the evolution of dendrimer structure as a function of external thermodynamical parameters is necessary.

There have been extensive research activities focused on understanding the molecular protonation of PAMAM dendrimers and its influence on their equilibrium structure. ${ }^{1,4}$ Simulation of polyelectrolyte dendrimers has been 
concerned with the dependence of single molecular structural properties, such as structural backfolding, global size of the dendrimer (in terms of the radius of gyration $R_{G}$ ), and the intra-molecular radial density profile of each constituent component such as terminal groups, water, and counterions, on molecular protonation. ${ }^{5-25} \mathrm{~A}$ series of small angle neutron scattering (SANS) investigations ${ }^{26-31}$ of charged PAMAM dendrimer solutions has clearly revealed that the backfolding of polymeric components, which was first proposed by Lescanec and Muthukumar, ${ }^{32}$ is a generic feature of polyelectrolyte dendrimers, regardless their molecular charge and generation. Their structural response to molecular charge is characterized by a transition in the intra-dendrimer density profile involving mass re-distribution from the central region to the molecular periphery. For both lower generation PAMAM dendrimers, with a more open molecular architecture and higher generation ones with significant steric hindrance, this conformational change is accompanied by a relatively minor electrostatic swelling. Moreover, the incoherent scattering function measured by the quasi-elastic neutron scattering (QENS) technique indicates that the local segmental dynamics is substantially enhanced in response to an increase of the electrostatic interaction. ${ }^{33}$ Contrast variation SANS has also been employed in our previous structural evaluation of dendrimer solutions. ${ }^{34}$ From the water accessible intra-dendrimer space, the porosity of neutral dendrimer as a function of generation was quantitatively evaluated. However, little is known about the spatial arrangement of constituent components of dendrimer molecules themselves. Their dependence on molecular charge and dendrimer generation remains unexplored experimentally as well.

Therefore, the purpose of this study, which belongs to a series of our experimental investigations of charged PAMAM dendrimer solutions, was to determine the intra-molecular radial distributions of intra-molecular penetrating water and the polymeric components of PAMAM dendrimers using contrast variation SANS. Based on the quantitative structural information obtained from model fitting, the evolution of intradendrimer hydration level and mass relocation were revealed for two generations of dendrimers with different molecular porosities. The relationship between the backfolding phenomenon and the molecular protonation was also evaluated.

This paper is organized as follows: Section II gives a brief discussion about sample preparation and synthesis of partially deuterated dendrimers. In Sec. III, the small angle scattering experimental procedures are described. In Sec. IV, the theoretical framework of the SANS data analysis is presented. Results of our data analysis are given in Sec. V. Special emphasis is given to discussing the spatial distributions of the constituent components of dendrimers and their dependence on the molecular protonation. We summarize this report in Sec. VI and present the small angle x-ray scattering (SAXS)/SANS study of the partially deuterated G5 dendrimer solution in the Appendix.

\section{MATERIALS}

Fully protonated, high purity biomedical grade Generation 4 and 7 (G4 and G7) polyamidoamine starburst den- drimers with ethylenediamine cores were purchased from Dendritech Inc., Midland, MI. ${ }^{35}$ Deuterium chloride and deuterium oxide $\left(\mathrm{D}_{2} \mathrm{O}\right)$ were acquired from Cambridge Isotope Laboratories, Inc., Andover, MA. The samples for the SANS experiments were prepared according to a previously developed procedure described in Ref. 26 . The dendrimer concentration was fixed at $0.0225 \mathrm{~g} / \mathrm{ml}(\sim 2 \mathrm{wt}$. \%). The acidity of the dendrimer solution is quantified by the parameter $\alpha$ defined as the molar ratio of acid to that of the primary amines present in the solution.

Isotopically labeled G5 PAMAM dendrimers with the inner three generational layers protonated and the outer two layers deuterated was synthesized at the Center for Nanophase Materials Sciences, Oak Ridge National Laboratory (ORNL) using a divergent, iterative approach that involves the repetitive application of a Michael addition followed by amidation. Details of the synthesis procedure and characterizations are described in Ref. 36. Again the partially deuterated dendrimers were dissolved in $\mathrm{D}_{2} \mathrm{O}$ with 2 wt. $\%$ concentration for scattering experiment.

\section{SMALL ANGLE NEUTRON AND X-RAY EXPERIMENTS}

SANS measurements were performed on the EQSANS spectrometer at the Spallation Neutron Source, ORNL, NG7 SANS at the NIST Center for Neutron Research (NCNR), National Institute of Standards and Technology (NIST), and 40m-SANS Spectrometer at High-flux Advanced Neutron Application Reactor (HANARO) at Korea Atomic Energy Research Institute (KAERI). The range of probed wave vector $Q$ was set to be from 0.01 to $0.5 \AA^{-1}$. The samples were contained in $1 \mathrm{~mm}$ path length Hellma quartz cells (category number 120, mat. Code QS). The experiments were carried out at ambient temperature and the measured SANS intensity was converted to absolute scale $\left(\mathrm{cm}^{-1}\right)$ with corrections accounting for background, detector sensitivity, and empty cell contribution based on standard procedure described in Ref. 37.

A SAXS experiment was conducted to explore the conformational dependence of partially deuterated G5 PAMAM dendrimers in $\mathrm{D}_{2} \mathrm{O}$ solution on the molecular protonation. The experiment was carried out at BL23A1 SAXS end station of the National Synchrotron Radiation Research Center (NSRRC), Taiwan. To avoid radiation damage of the dendrimer structure caused by high energy $\mathrm{x}$ rays, a rocking cell was used to accommodate the samples during the experiment, and the data collection time for each sample was $50 \mathrm{~s}$. The absolute intensity was calibrated by a high density polyethylene (HDPE) standard. The SAXS profiles were corrected for background scattering, sample transmission, empty cell transmission, empty cell scattering, and the detector sensitivity.

\section{THEORETICAL BASIS OF SANS DATA ANALYSIS}

The measured SANS absolute intensity $I(Q)$ can be modeled by the following integral equation which incorporates the theoretical coherent scattering cross section $I_{t h}(Q)$ and the 
instrument resolution function:

$$
I(Q)=\int \frac{I_{t h}(Z)}{\sqrt{2 \pi \delta(Q)^{2}}} \exp \left[-\frac{\left(Z-Q_{m}\right)^{2}}{2 \delta(Q)}\right] d Z .
$$

In Eq. (1), the instrument resolution is approximated by a Gaussian function with standard deviation $\delta(Q)$ centered at $Q_{m}$, which are provided at each instrument at the time of data acquisition.

Assuming a spherical shape for the PAMAM dendrimer and low monodispersity, the $I_{t h}(Q)$ in Eq. (1) for an interacting dendrimer solution can be conveniently factorized into the following expression: ${ }^{38}$

$$
I_{t h}(Q)=A P(Q) S(Q)+I_{I N C},
$$

where $A$ gives the scattering amplitude which is a function of the scattering contrast between dendrimer and the surrounding aqueous solvent, dendrimer number density, and the dendrimer average molecular volume. Here, $P(Q)$ the normalized form factor, or intra-dendrimer structure factor, contains the information of the intra-particle density profile, $S(Q)$ the interparticle structure factor, describes the spatial arrangement of dendrimers, and $I_{I N C}$ is the incoherent background. In this study, the dendrimer molecule is modeled as a centrosymmetric spherical object with an effective hard-core radius $R$ and a diffusive molecular periphery whose fuzziness is characterized by a parameter $\sigma .{ }^{26}$ The corresponding $S(Q)$ is obtained by numerically solving the Ornstein-Zernike equation with hypernetted chain closure. ${ }^{39}$ The experimental data are analyzed using a MATLAB ${ }^{35}$ program and details can be found in Ref. 26.

The scattering amplitude A in Eq. (2) takes the following analytical form:

$$
\begin{aligned}
A & =n_{s}\left(\Delta \rho \times v_{\text {dendrimer }}\right)^{2} \\
& =n_{s}\left[\left(\rho_{\text {dendrimer }}-\rho_{\text {sovlent }}\right) \times v_{\text {dendrimer }}\right]^{2},
\end{aligned}
$$

where $n_{s}$ is the number density of dendrimers, $\rho_{\text {dendrimer }}$ and $\rho_{\text {solvent }}$ are the average scattering length densities (SLDs) of a dendrimer and a solvent, respectively, and $v_{\text {dendrimer }}$ is the dendrimer volume. If one defines $\gamma$ as the volume fraction ratio of $\mathrm{D}_{2} \mathrm{O}$ in the solvent, $\rho_{\text {solvent }}$ can be further expressed as

$$
\rho_{\text {sovlent }}=\gamma \rho_{\mathrm{D}_{2} \mathrm{O}}+(1-\gamma) \rho_{\mathrm{H}_{2} \mathrm{O}},
$$

where $\rho_{\mathrm{D}_{2} \mathrm{O}}$ and $\rho_{\mathrm{H}_{2} \mathrm{O}}$ are the scattering length densities of $\mathrm{D}_{2} \mathrm{O}$ and $\mathrm{H}_{2} \mathrm{O}$, respectively. $\gamma$ ranges from 0 (pure $\mathrm{H}_{2} \mathrm{O}$ ) to 1 (pure $\mathrm{D}_{2} \mathrm{O}$ ).

It is important to note that the total neutron SLD distribution $\rho_{\text {dendrimer }}(r)$ is indeed a collective manifestation of the SLD distribution of polymeric constituent components of the dendrimer, $\rho_{\text {polymer }}(r)$, and the intra-dendrimer penetrating water distribution $\rho_{\text {solvent }}(r)$, which is determined by the water distribution $H(r)$ and labile proton distribution $n_{e x}(r)$ along the radial direction. In other words, $\rho_{\text {dendrimer }}(r)$ can be analytically expressed as

$$
\begin{aligned}
\rho_{\text {dendrimer }}(r)= & \rho_{\text {polymer }}(r)+b_{\text {solvent }} H(r)+n_{\text {ex }}(r) \\
& \times\left[\gamma b_{D}+(1-\gamma) b_{H}\right],
\end{aligned}
$$

where $b_{D}$ and $b_{H}$ are the scattering lengths of deuterium and hydrogen atoms, respectively, and $b_{\text {solvent }}$ is the average scattering length of the solvent,

$$
b_{\text {sovlent }}=\gamma b_{\mathrm{D}_{2} \mathrm{O}}+(1-\gamma) b_{\mathrm{H}_{2} \mathrm{O}}=\rho_{\text {sovlent }} v_{\text {water }},
$$

with the molecular volume of water $v_{\text {water }}=30 \AA^{3}$.

The essence of this SANS data analysis is to determine $\rho_{\text {polymer }}(r), H(r)$, and $n_{e x}(r)$, as explicitly contained in Eq. (5), from the variation of SANS intensity distribution as a function of $\gamma$.

\section{RESULTS AND DISCUSSION}

Figure 1 presents the SANS absolute intensity distributions $I(Q)$ obtained from the aqueous solutions of $2 \mathrm{wt}$. \% G4 and G7 fully protonated PAMAM dendrimers and the corresponding model fitting curves. It is first noticed that upon changing the molecular protonation from $\alpha=0$ (Fig. 1(a) for G4 and Fig. 1(c) for G7) to $\alpha=1$ (Fig. 1(b) for G4 and Fig. 1(d) for G7), enhanced local ordering, due to the inter-dendrimer Coulomb repulsion, is reflected in SANS interaction peak observed in the region of $Q<0.1 \AA^{-1}$. Second, the scattering contrast between the dendrimer molecules and surrounding solvent is seen to reduce monotonically with the increase in the molar ratio of $\mathrm{H}_{2} \mathrm{O}$. The magnitude of the SANS $I(Q)$ corresponding to the sample with higher $\gamma$ value is seen to be more than that of the sample with lower $\gamma$ value. The detailed qualitative features of the SANS $I(Q)$, including the interaction peak and the discernible bump located between $Q=0.1$ and $0.2 \AA^{-1}$, are gradually masked by the progressive increase of the incoherent scattering introduced by the increasing amount of protons in the aqueous solvent. As a consequence, despite the generally good agreement between the experimental $I(Q)$ and the theoretical model of scattering cross section for dendrimer shown in Fig. 1, the statistical uncertainties of the structural characteristics for dendrimer, such as the effective hard core radius $R$ and $\sigma$ the parameter characterizing the molecular softness, inevitably increase with the decrease in $\gamma$, in both the neutral state $(\alpha=0)$ and charged state $(\alpha=1)$. In order to minimize the uncertainty of the structural information, such as intra-dendrimer hydration level and mass distribution of polymeric components extracted from SANS model fitting, the conformational characteristics of single dendrimer molecules $R$ and $\sigma$ were first determined from the $I(Q)$ corresponding to the dendrimers dissolved in $100 \% \mathrm{D}_{2} \mathrm{O}$ solution (the red symbols in Figs. 1(a)-1(d)). $R$ and $\sigma$ were then fixed in the SANS data analysis for samples with varying $\gamma$ values. By varying the hydrogen-to-deuterium (H/D) ratio in the solvent, the goal of the SANS data analysis is to compartmentalize the contribution of water molecules from that of polymer component and associated chlorides in the measured scattering cross section. If the spatial distribution of polymer component and counterions does not depend on the H/D ratio of aqueous solvent, the spatial distribution of water along the radial direction of dendrimer molecule can be conveniently deduced from the normalized excess density profiles $\Delta \rho_{\text {dendrimer }}(r)$ (Fig. 2$)$ which can be calculated from the extracted $R$ and $\sigma$ for the case of $100 \% \mathrm{D}_{2} \mathrm{O}$. For $\mathrm{G} 4 \alpha=0: R=19.81 \AA, \sigma=10.95 \AA$ and $\alpha=1$ : 


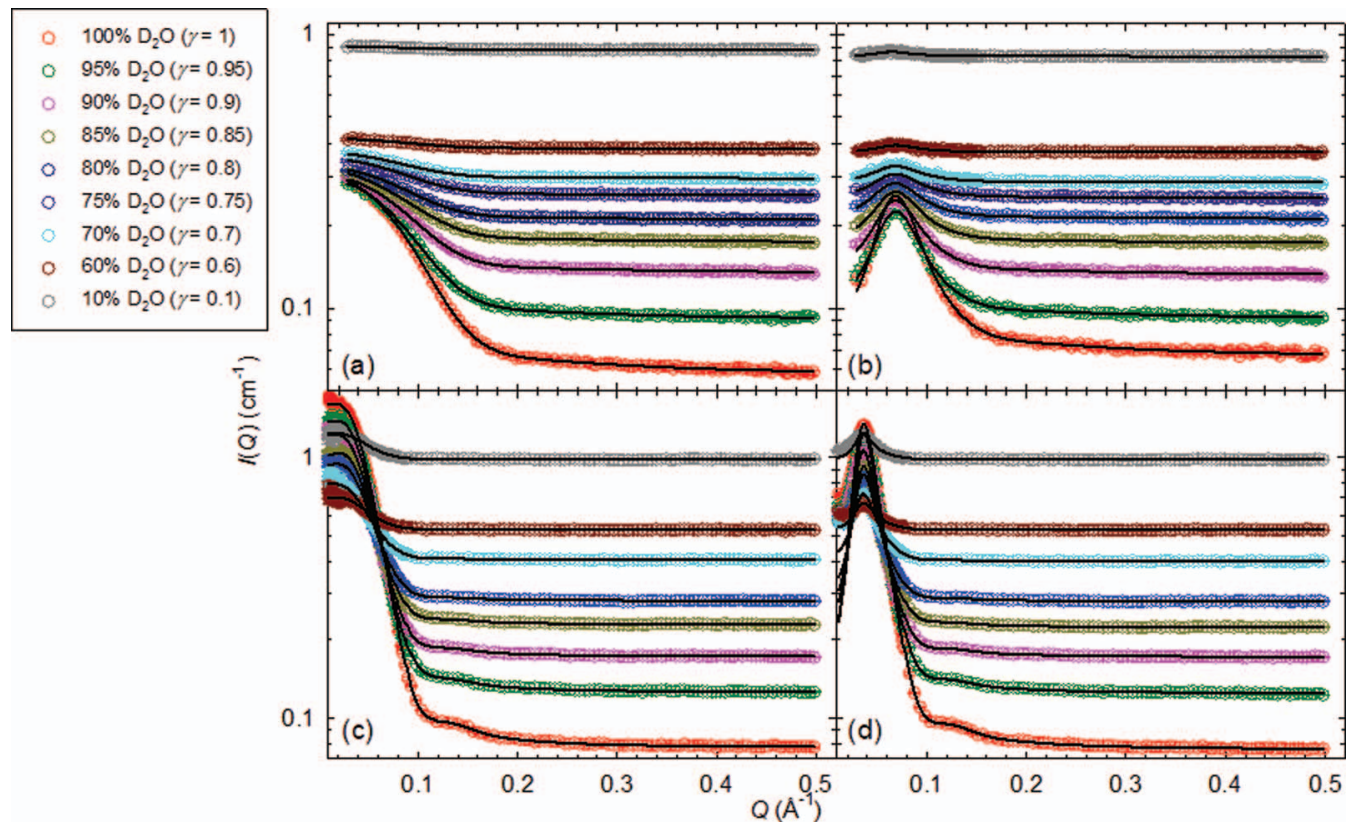

FIG. 1. The SANS absolute intensity $I(Q)$ and the associated model fitting curves of $\mathrm{G} 4$ and $\mathrm{G} 7$ dendrimer solutions with varying volume fraction of $\mathrm{D}_{2} \mathrm{O}(\gamma)$. Panel (a) presents the $I(Q)$ for G4 dendrimer solution of $\alpha=0$, (b) for G4 dendrimer solution of $\alpha=1$, (c) for G7 dendrimer solution of $\alpha=0$, and (d) for G7 dendrimer solution of $\alpha=1$. Different colors are used to specify the H/D ratio of the solvent. The good quantitative agreement between the experiment and model is clearly seen. The magnitude of experimental uncertainties is less than that of the symbol size.

$R=22.04 \AA, \sigma=10.6 \AA$; for $\mathrm{G} 7 \alpha=0: R=39.57 \AA$ $=12.6 \AA$ and $\alpha=1: R=43.76 \AA, \sigma=12.45 \AA$. For the case of the neutral dendrimer based on Eq. (5), $\Delta \rho_{\text {dendrimer }}(r)$ includes the contributions from the polymeric component which does not change with the contrast, the associated water molecules inside the dendrimer, and the labile protons. The expression is found to be

$$
\begin{aligned}
\Delta \rho_{\text {dendrimer }}(r)= & \rho_{\text {polymer }}(r)+b_{\text {solvent }}\left[H(r)-\frac{1}{30}\right] \\
& +n_{e x}(r) \times\left[\gamma b_{D}+(1-\gamma) b_{H}\right] .
\end{aligned}
$$

The purpose of preparing dendrimer solutions with same concentration and level of molecular protonation but nine

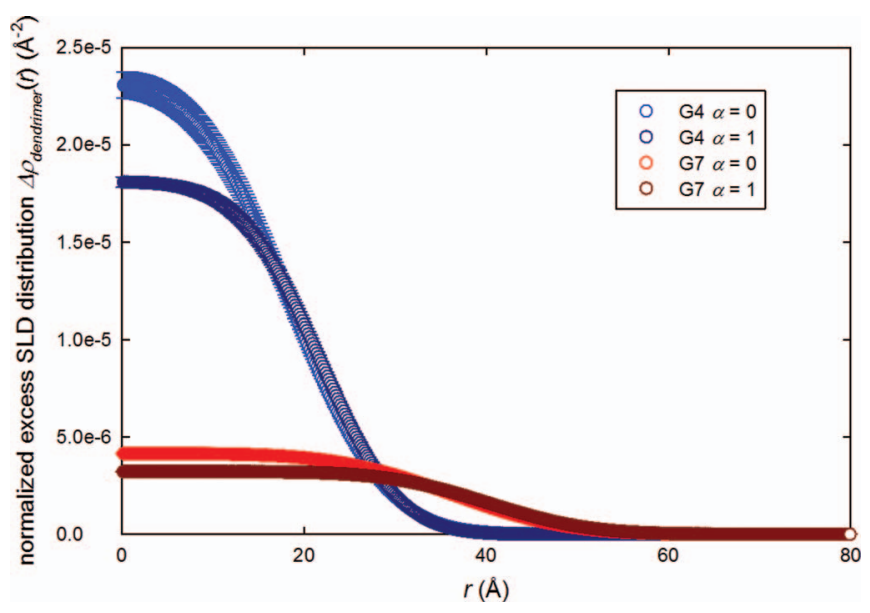

FIG. 2. The normalized excess neutron scattering length density profiles $\Delta \rho_{\text {dendrimer }}(r)$ for G4 and G7 PAMAM dendrimers obtained neutral $(\alpha=0)$ and charged $(\alpha=1)$ states. different $\gamma$ values for SANS experiment is to minimize the uncertainties of extracted spatial distribution of each component. This scheme can be clearly seen from Eq. (5) in Sec. IV.

Before discussing the results of our data analysis, it is instructive to comment on the normalized excess SLD profiles presented in Fig. 2, which are collective manifestations of the total contribution of each constituent component of dendrimer solution. As defined in our previous reports, the normalized density distribution renders the SLD distribution of the probed dendrimer system along the radial direction. A noticed feature of Fig. 2 is that for both G4 and G7 PAMAM dendrimers, the overall SLD distributions are seen to exhibit their maximum at the center of the dendrimer and decay toward the molecular periphery. There have been extensive discussions about the connection between the intra-dendrimer mass profile and its unique molecular architecture. ${ }^{1,2}$ Our observation presented in Fig. 2 clearly supports the so-called "dense-core" picture, ${ }^{32}$ originating from backfolding of the polymeric components of the dendrimer. An experimental study of isotopically labeled G5 PAMAM dendrimer using SANS and SAXS also reaches the same conclusion, and the results are presented in the Appendix. Second, the SLD distribution of G4 PAMAM dendrimer (blue and dark blue curves) is seen to be more localized around the molecular central region, in comparison to that of G7 PAMAM dendrimer (red and dark red curves). This difference reflects the fact that due to the increasing steric excluded-volume effect, PAMAM dendrimers of higher generation indeed have more uniform intra-molecular mass distribution in comparison to that for lower generation dendrimers. Moreover, upon increasing the molecular protonation, an outward mass redistribution, indicated by the decrease of the normalized density distribution in the molecular central region and the increase in the molecular peripheral region, 


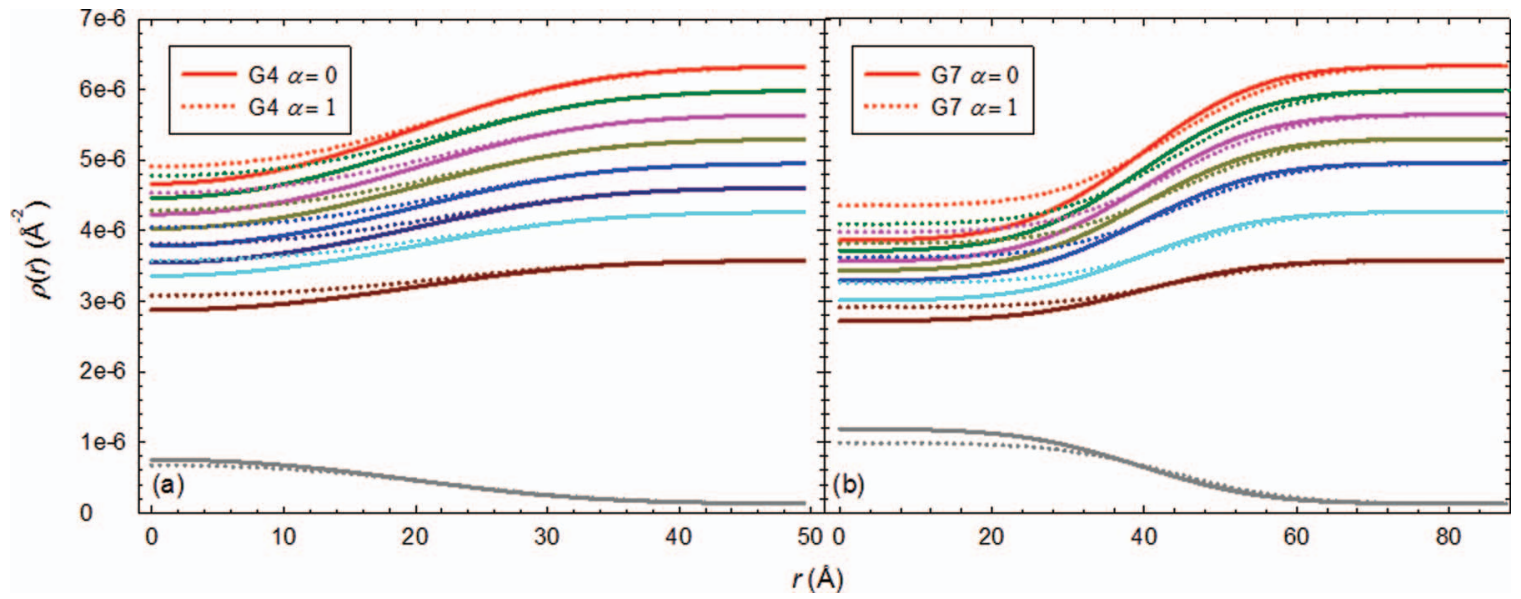

FIG. 3. The evolution of total scattering length density distribution for G4 (a) and G7 (b) PAMAM dendrimers as a function of H/D ratio of the aqueous solvent. The color scheme used in this figure is identical to that of Fig. 1.

is clearly seen in both generations of PAMAM dendrimers. This observation provides compelling evidence that entropytriggered backfolding is indeed a generic feature of PAMAM dendrimers dissolved in aqueous solvent, independent of enhancement of steric hindrance with advancing dendrimer generation.

The various dendrimer scattering length density distributions $\rho_{\text {dendrimer }}(r)$ revealed by SANS model fitting as a function of $\gamma$ are presented in Fig. 3. The solids curves represent the neutral case $(\alpha=0)$ and the dotted ones for the charged case $(\alpha=1)$. The choice of colors representing the $\gamma$ values of the probed samples is identical to that used in Fig. 1. As $r$ approaches infinity, each curve in Fig. 3 is seen to approach its own asymptotic value. From Eq. (5) it can be easily seen that the value of $\rho_{\text {dendrimer }}(r \rightarrow \infty)$ is simply $b_{\text {solvent }} H(r)$, the second term present on the right-hand side (RHS) of Eq. (5). For bulk water, assuming there is no isotopic effect, $H(r)$ $=v_{\text {water }}{ }^{-1}=0.03 \AA^{-3}$. Therefore, $\rho_{\text {dendrimer }}(r \rightarrow \infty)$ is equal to $0.03 b_{\text {solvent }} \AA^{-2}$ and, therefore, a linear function of $\gamma$, as indicated by Eq. (6). As $r$ decreases and approaches the periphery of the dendrimer molecule, the calculated $\rho_{\text {dendrimer }}(r)$ for neutral dendrimers ( $\alpha=0$, solid lines) is seen to vary characteristically due to the additional scattering contributions from the polymeric component of the dendrimer (first term on the RHS of Eq.(5)) and the compositional effect of liable protons in the dendrimer (third term on the RHS of Eq.(5)). For the charged case $(\alpha=1)$, additional contribution from the spatial arrangement of counterions (chlorides) needs to be incorporated into the expression of $\rho_{\text {dendrimer }}(r)$, namely,

$$
\begin{aligned}
\rho_{\text {dendrimer }}(r)= & \rho_{\text {polymer }}(r)+b_{\text {solvent }} H(r)+n_{\text {ex }}(r) \\
& \times\left[\gamma b_{D}+(1-\gamma) b_{H}\right]+b_{\text {chloride }} C(r),
\end{aligned}
$$

where $b_{\text {chloride }}$ is the bound scattering length of chloride and $C(r)$ is the radial distribution function of chlorides in the solvent. It is important to note that for the charged case, $\rho_{\text {polymer }}(r)$ and $H(r)$ may be different from their neutral counterparts because of the structural distortion resulting from the arrangement of the positively charged amines and the associ- ated chlorides which are oppositely charged. Therefore, the observed difference between the solid lines and the dotted ones for a given $\gamma$ value cannot be simply attributed to the contribution from $b_{\text {chloride }} C(r)$ in Eq. (8).

Based on the aforementioned approximation of negligible isotope effect, one can determine $H(r)$ for the case of $\alpha$ $=0$ and $\alpha=1$ from Eqs. (5) and (7), respectively, and the results are presented in Fig. 4(a) (for G4) and Fig. 4(b) (for G7). In both cases, $H(r)$ is seen to increase within the molecular central region when $\alpha$ increases from 0 to 1 . For the case of G7, a discernible decrease in $H(r)$ around the molecular periphery is further identified within the experimental errors. As repeatedly predicted by numerous simulations of dendrimers, a conformational change from a more diffusive dense-core profile to a more uniformly distributed one is triggered by molecular protonation of dendrimer. ${ }^{1}$ This outward stretching of polymeric components creates additional intra-dendrimer space which is accessible to the penetrating water molecules and this observation is, therefore, consistent with general intuitive expectation. A second feature revealed in our data analysis is the structural openness of the lower generation dendrimer: The effect of steric crowding can be clearly visualized from the magnitude of $H(r)$ of G4 and G7 dendrimers around the molecular central region for both case of $\alpha=0$ and $\alpha$ $=1$. Figs. 4(c) and 4(d) give the total number of penetrating water $n_{\text {water }}(r)$ confined within a sphere which centers at the molecular center of the dendrimer for G4 and G7 dendrimers, respectively. Assuming the spatial distribution of intradendrimer water is spherically symmetric, the mathematical relation between $H(r)$ and $n_{\text {water }}(r)$ is given by the following expression:

$$
n_{\text {water }}(r)=\int_{0}^{r} 4 \pi l^{2} H(l) d l,
$$

where $l$ is a dummy variable of integration. Contrary to the charge dependence of $n_{\text {water }}(r)$ for G7 dendrimer, within experimental uncertainty, no difference is evident between the $n_{\text {water }}(r)$ corresponding for $\alpha=0$ and that for $\alpha=1$ for the case of G4. This observation proves that the steric hindrance indeed becomes progressively significant with an increase in dendrimer generation. The results shown in Fig. 4 are 


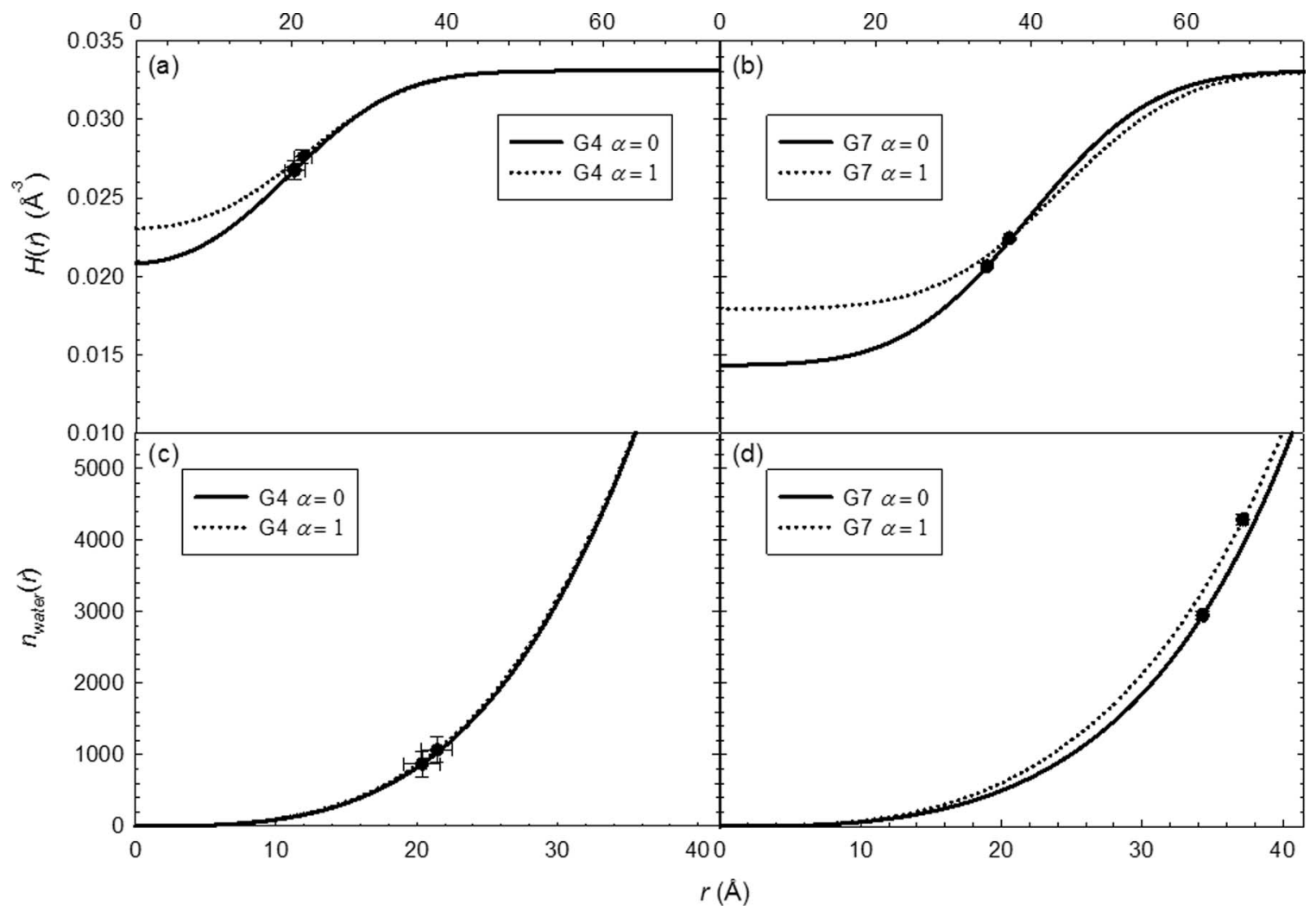

FIG. 4. The number density of penetrating water per unit volume and total number of penetrating water for G4 ((a) and (c)) and G7 ((b) and (d)) as a function of molecular protonation.

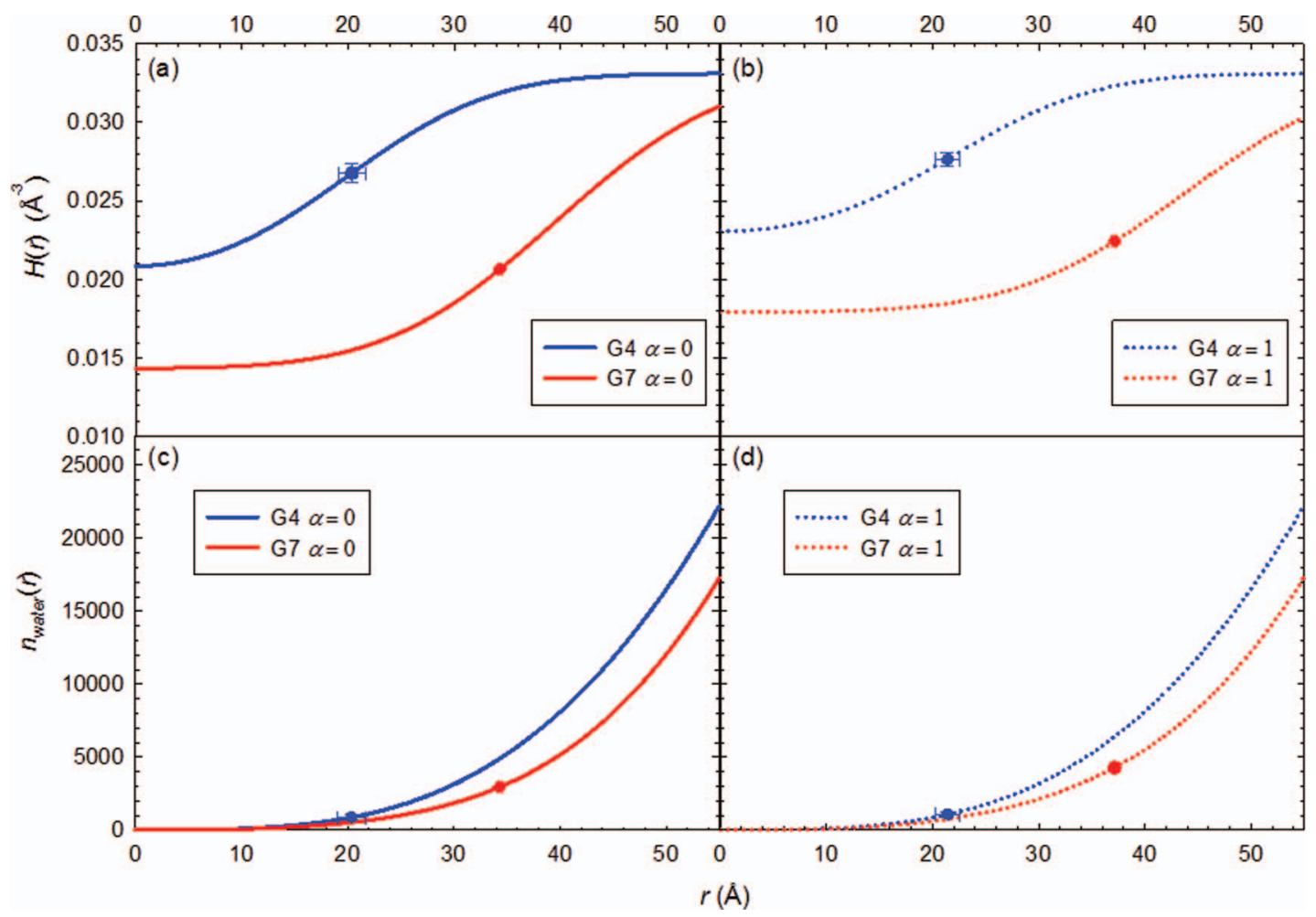

FIG. 5. Rearrangement of the results presented in Fig. 4 in a comparative manner of dendrimer generation. 
re-arranged in a comparative manner for G4 and G7 dendrimers as shown in Fig. 5. The filled circles in Figs. 4 and 5 identify the values of water number density and total number at the distance of the radius of gyration of the dendrimer, and $R_{G}$ is calculated based on the approach developed previously. $^{26}$ Indeed, the intra-dendrimer free space which is accessible to water molecules is seen to significantly decrease due to the higher segmental density in the G7 PAMAM dendrimer, irrespective of the molecular protonation. However, the evolving $H(r)$ and $n_{\text {water }}(r)$ as a function of molecular protonation for G7 again unambiguously demonstrate the structural flexibility of higher generation PAMAM dendrimer and, therefore, the existence of polymer backfolding, even with the presence of substantial steric crowding. Moreover, the increase in intra-dendrimer porosity due to molecular protonation also provides an experimental support for the charge-triggered enhancement in individual segmental motion observed by the QENS experiment. ${ }^{33}$ It was our conjecture that the introduction of intra-molecular charge to some extent relieves the steric crowding and creates extra local space available for local segmental motion driven by thermal energy. Judging from the results shown in Fig. 5, the higher hydration level for charged dendrimers suggests the molecular protonation indeed increases the molecular porosity and, therefore, qualitatively justifies our previous hypothesis. This conjecture is also consistent with the conclusion of NMR experiment conducted by Meltzer and coworkers. ${ }^{40}$

Subtracting the intra-dendrimer water distribution presented in Fig. 4 from the total scattering length density distribution shown in Fig. 2, the SLD distribution of which does not vary with the solvent contrast, including contributions from both the polymeric component and counterions, as a function of $r$ can be revealed and the results are presented in Fig. 6 . Due to the additional Coulombic repulsion among the charged amines, although severely screened by highly localized associated chlorides, in both cases of G4 and G7 the distribution SLD is seen to shift toward the molecular periphery. Moreover, as a consequence of a higher polymeric segmental density, the SLD at any given $r$ for G7 is seen to be higher than that for G4, which is consistent with the intra-dendrimer hydration level presented in Figs. 4 and 5.
One major prediction of numerous dendrimer simulation studies is the intra-dendrimer counterion distribution and it is, therefore, intriguing to explore the possibility of experimentally further identifying the contribution of associated counterions to the SLD distribution from that of the polymeric components. In other words, attempts have been made to separate the contributions from the first and last terms on the RHS of Eq. (7) to the $\rho(r)$ for charged cases (dotted curves in Fig. 6), providing the detailed information of $H(r)$. From Eq. (7), it is clearly seen that in order to achieve this goal, quantitative information of $C(r)$ is required. One plausible scheme to evaluate the intra-dendrimer spatial distribution of associated chlorides from the polymer background is to take advantage of the difference of neutron and x-ray scattering cross sections for each relevant element of the probed dendrimer system. Accordingly, a complementary SAXS experiment with the same set of samples used in the SANS measurement was conducted. However, via this approach, resolving the intra-dendrimer spatial distribution of associated chlorides is severely compounded by the following intrinsic constraints: Although the neutron and x-ray cross sections for each element present in the polymeric skeleton are known, the backfolding phenomenon proved to be ubiquitous for dendrimers dissolved in aqueous solvent regardless of their generation or molecular protonation, introducing a fluctuation in the SLD distribution which masks the SLD variation caused by the associated chlorides. It is, therefore, concluded that, without an a priori mathematical model, it is not possible to quantitatively map out the intra-dendrimer chloride distribution based on the method proposed in this work.

\section{CONCLUSIONS}

In this paper, the structural characteristics of $\mathrm{G} 4$ and $\mathrm{G} 7$ PAMAM dendrimers dissolved in aqueous solutions were investigated by a contrast variation SANS technique. The focus was placed on understanding the connection between the spatial distributions of water and polymeric components of dendrimer, and the molecular protonation. By varying the hydrogen-to-deuterium ratio in the solvent, the intramolecular water and polymer/counterion distributions along

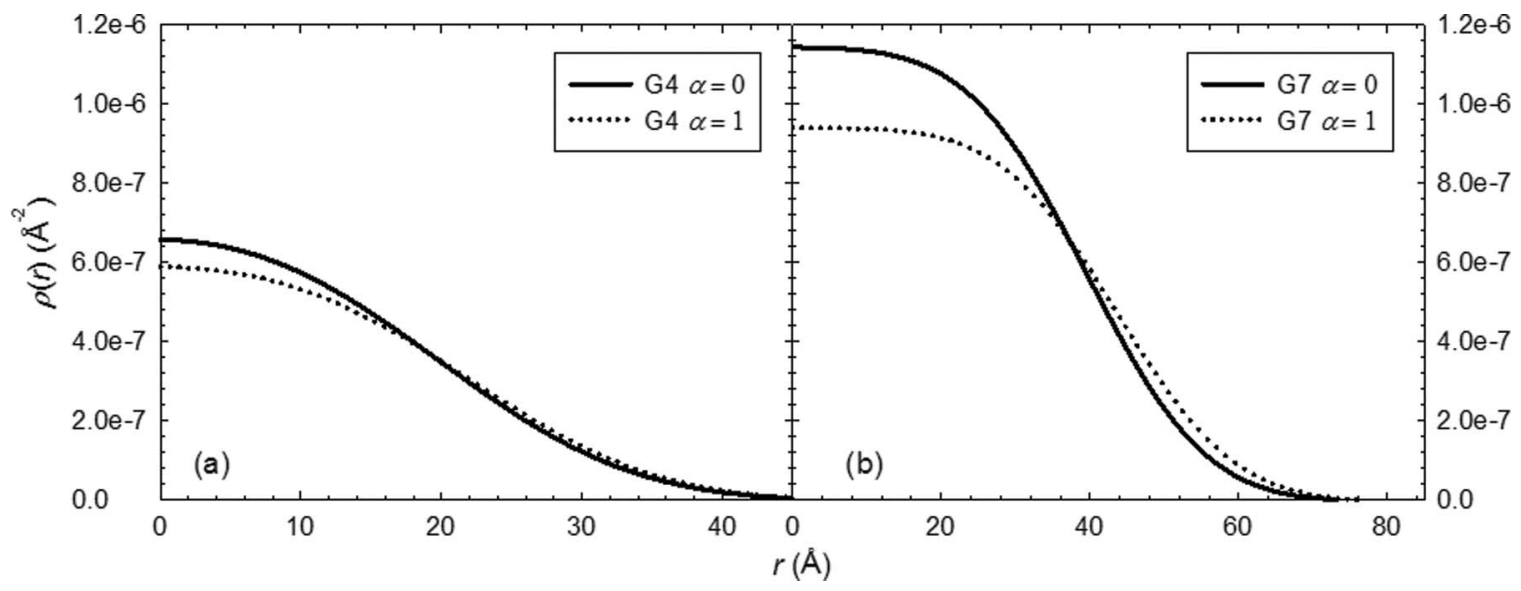

FIG. 6. The radial SLD distributions of polymer (solid lines, $\alpha=0$ ), and polymer and counterion (dotted lines, $\alpha=1$ ) for G4 (a) and G7 (b) PAMAM dendrimers. 

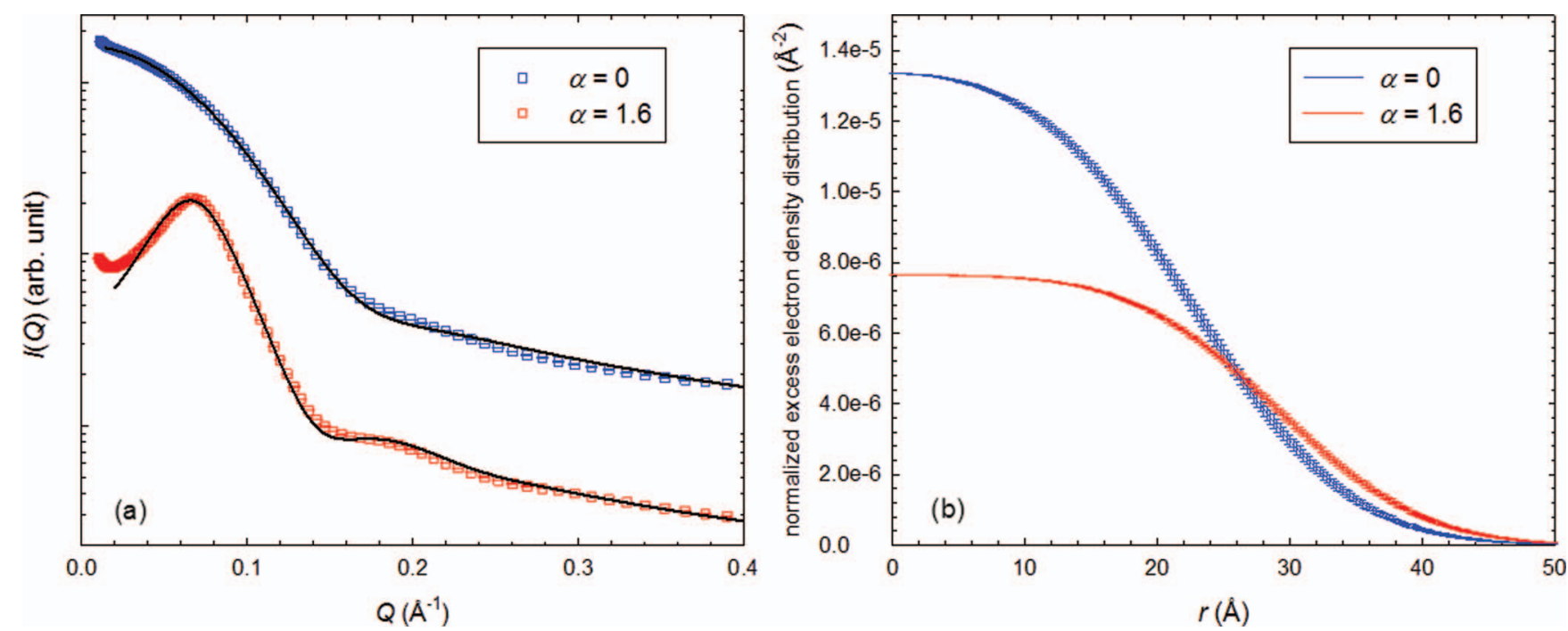

FIG. 7. (a) The SAXS spectra and corresponding model fitting curves for $2 \mathrm{wt} \% \mathrm{D}_{2} \mathrm{O}$ solutions of isotopically labeled G5 PAMAM dendrimers. (b) The calculated normalized excess electron density profiles.

the radial direction were compartmentalized from the total scattering length density distribution within the SANS accessible length scale based on the theoretical method described in this study.

Upon increasing the molecular protonation, the degree of hydration, namely, the number density of water per unit volume, is seen to increase within the molecular region for both cases of G4 and G7 PAMAM dendrimers. Meanwhile, an outward mass redistribution, in terms of the average SLD of polymeric components and counterions, is also revealed. Several important conclusions are drawn from our data analysis: First of all, by varying the molecular protonation and modeling the evolving spatial distributions of intra-dendrimer penetrating water and polymer components, we clearly demonstrate the existence of segmental backfolding. Second, along with the findings of the SAXS/SANS investigation of partially deuterated G5 PAMAM dendrimer, the evolution of mass re-distribution revealed by the contrast variation SANS

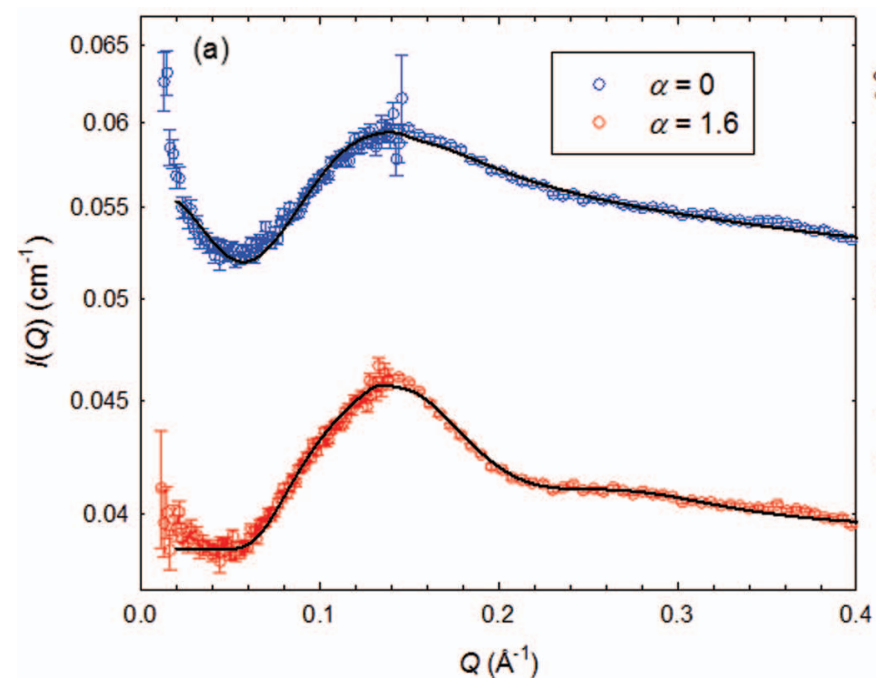

experiments suggests that PAMAM dendrimer indeed has a dense-core molecular density profile when dissolved in aqueous solutions. Moreover, the intra-molecular porosity is quantitatively revealed as a function of dendrimer generation. The connection between its dependence on molecular protonation and previously observed enhanced segmental dynamics is further explored.

\section{ACKNOWLEDGMENTS}

This Research at Oak Ridge National Laboratory's Spallation Neutron Source was sponsored by the Scientific User Facilities Division, Office of Basic Energy Sciences, U.S. Department of Energy. The research carried out at the Center for Nanophase Materials Sciences, Oak Ridge National Laboratory, was sponsored by the Scientific User Facilities Division, Office of Basic Energy Sciences, U.S. Department of Energy. This SANS experiment utilized facilities supported in part

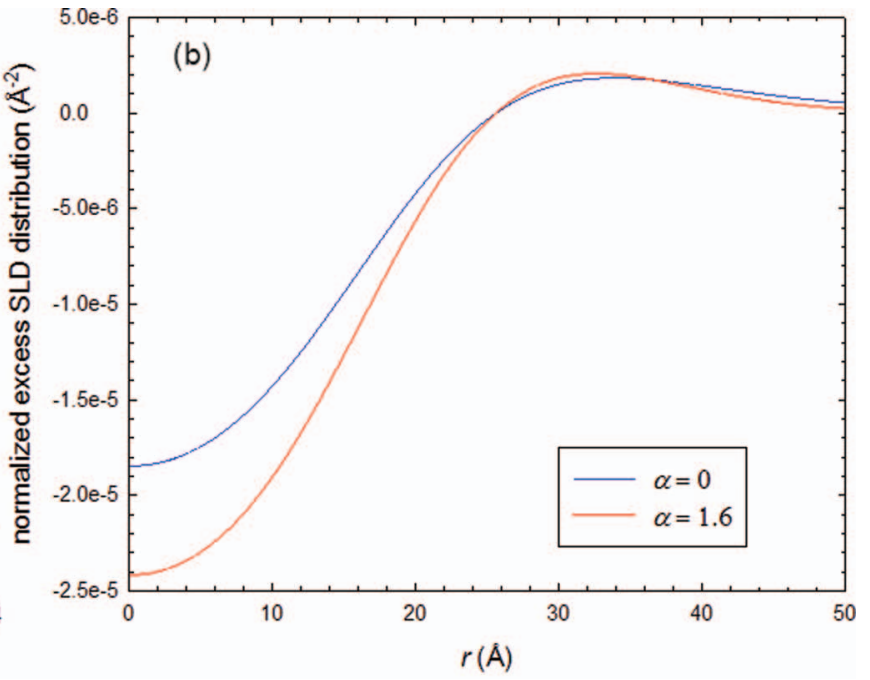

FIG. 8. (a) The SANS spectra and corresponding model fitting curves for 2 wt. $\% \mathrm{D}_{2} \mathrm{O}$ solutions of isotopically labeled G5 PAMAM dendrimers. (b) The calculated normalized excess SLD profiles. 
by the National Science Foundation under Agreement No. DMR-0944772. We greatly appreciate the SANS beam time from HANARO KAERI, Korea, and SAXS beam time from NSRRC, Taiwan as well as the technical supports from both facilities for our experiment. B.W., X.L., and E.L.L. acknowledge the financial support by U.S. Department of Energy under NERI-C Award No. DE-FG07-07ID14889 and U.S. Nuclear Regulatory Commission under Award No. NRC-38-08950 .

\section{APPENDIX: STRUCTURAL INVESTIGATION OF G5 ISOTOPICALLY LABELED PAMAM DENDRIMERS DISSOLVED IN $\mathrm{D}_{2} \mathrm{O}$ USING SAXS AND SANS TECHNIQUES}

To explore the intra-dendrimer density profile and its dependence on molecular protonation, an experimental study using SAXS and SANS was employed to investigate the partially deuterated G5 PAMAM dendrimers with protonated central region (G0-3) and deuterated outer shell (G4-5). Figure 7(a) presents the SAXS experimental results and fitting curves based on the model proposed in our previous reports. Similar to the SANS experiment of fully protonated G5 dendrimers, ${ }^{26}$ the normalized excess electron density profile presented in Fig. 7(b) exhibits a transition from a more densecore picture at the neutral state to a more uniform hard spherelike profile when the molecular protonation is increased to the level of $\alpha=1.6$.

The results obtained from the corresponding SANS experiment are presented in Fig. 8(a). The SANS coherent scattering cross sections $I(Q)$ are seen to be intrinsically different from existing spectra of fully protonated G5 dendrimer solutions due to the difference of neutron scattering cross section for hydrogen and deuterium. The SLD of the deuterated molecular periphery is found to be slightly higher than that of the surrounding $\mathrm{D}_{2} \mathrm{O}$, while the SLD of protonated interior remains negative. When dissolved in $\mathrm{D}_{2} \mathrm{O}$, the molecular design of this partially deuterated G5 dendrimer greatly offsets the coherent neutron scattering and as a result, the main characteristics of the SANS and SAXS spectra, such as the interaction peak of $I(Q)$ centered at $Q=0.07 \AA^{-1}$ for the charged fully protonated G5 PAMAM dendrimers dissolved in $\mathrm{D}_{2} \mathrm{O}(\alpha=1.6)$, are no longer observed in the SANS $I(Q)$ presented in Fig. 8(a). This arises as $I(Q)$ is the product of $P(Q)$ and $S(Q)$ and the interaction peak in $S(Q)$ is suppressed by the specific distribution of $P(Q)$ for the partially deuterated dendrimer. Therefore, the $S(Q)$ obtained from SAXS data analysis of charged $\mathrm{G} 5 \mathrm{D}_{2} \mathrm{O}$ solution was used as the input to our SANS data analysis to incorporate the concentration effect. Within the protonated molecular central region $(r<16$ $\AA$ estimated based on our previous SANS study of fully protonated G3 PAMAM dendrimer solutions), a significant decrease of SLD is revealed in the normalized excess SLD profiles presented in Fig. 8(b). Considering the enhancement of SLD provided by the invasive $\mathrm{D}_{2} \mathrm{O}$ in the same region (similar to the picture presented in Fig. 4) which partially offsets the magnitude of decrease, the relocation of the deuterated polymeric components from the molecular central region to periphery by charged amines can be easily visualized and is consistent with the intra-molecular structural conformation revealed by SANS for G4 and G7 in the main body of this manuscript. This observation of structural backfolding again provides compelling evidence for the dense-core molecular mass density profile.

${ }^{1}$ M. Ballauff and C. N. Likos, Angew. Chem., Int. Ed. 43, 2998 (2004).

${ }^{2}$ C. N. Likos, Soft Matter 2, 478 (2006).

${ }^{3}$ C. C. Lee, J. A. MacKay, J. M. J. Fréchet, and F. C. Szoka, Nat. Biotechnol. 23, 1517 (2005).

${ }^{4}$ D. Astruc, E. Boisselier, and C. Omelas, Chem. Rev. 110, 1857 (2010).

${ }^{5}$ P. Welch and M. Muthukumar, Macromolecules 31, 5892 (1998).

${ }^{6}$ I. Lee, B. D. Athey, A. W. Wetzel, W. Meixner, and J. R. Baker, Macromolecules 35, 4510 (2002).

${ }^{7}$ P. K. Maiti, T. Çağun, S.-T. Lin, and W. A. Goddard, Macromolecules 38, 979 (2005).

${ }^{8}$ H. Lee, J. R. Baker, Jr., and Ronald G. Larson, J. Phys. Chem. B 110, 4014 (2006).

${ }^{9}$ P. K. Maiti and W. A. Goddard, J. Phys. Chem. B 110, 25628 (2006).

${ }^{10}$ A. W. Opitz and N. J. Wagner, J. Polym. Sci., Part B: Polym. Phys. 44, 3062 (2006)

${ }^{11}$ G. Giupponi, D. M. A. Buzza, and D. Adolf, Macromolecules 40, 5959 (2007).

${ }^{12}$ K. Karatasos, Macromolecules 41, 1025 (2008).

${ }^{13}$ R. Blaak, S. Lehmann, and C. N. Likos, Macromolecules 41, 4452 (2008).

${ }^{14}$ J. J. Freire, Soft Matter 4, 2139 (2008).

${ }^{15}$ J. S. Kłos and J.-U. Sommer, Macromolecules 42, 4878 (2009).

${ }^{16}$ Y. Liu, V. S. Bryantsev, M. S. Diallo, and W. A. Goddard, J. Am. Chem. Soc. 131, 2798 (2009)

${ }^{17}$ I. Tanis and K. Karatasos, J. Phys. Chem. B 113, 10984 (2009).

${ }^{18}$ W. Tian and Y. Ma, J. Phys. Chem. B 113, 13161 (2009).

${ }^{19}$ S. Huißmann, A. Wynveen, C. N. Likos, and R. Blaak, J. Phys.: Condens. Matter 22, 232101 (2010)

${ }^{20}$ S. Huißmann, C. N. Likos, and R. Blaak, J. Mater. Chem. 2010486 (2010).

${ }^{21}$ W. Tian and Y. Ma, Soft Matter 6, 1308 (2010).

${ }^{22}$ J. S. Kłos and J.-U. Sommer, Macromolecules 43, 4418 (2010).

${ }^{23}$ J. S. Kłos and J.-U. Sommer, Macromolecules 43, 10659 (2010).

${ }^{24}$ H. Lee and Ronald G. Larson, Macromolecules 44, 2291 (2011).

${ }^{25}$ S. V. Lyulin, L. J. Evers, P. van der Schoot, A. A. Darinskii, A. V. Lyulin, and M. A. J. Michels, Macromolecules 37, 3049 (2004).

${ }^{26}$ W.-R. Chen, L. Porcar, Y. Liu, P. D. Butler, and L. J. Magid, Macromolecules 40, 5887 (2007).

${ }^{27}$ L. Porcar, Y. Liu, R. Verduzco, K. Hong, P. D. Butler, L. J. Magid, G. S. Smith, and W.-R. Chen, J. Phys. Chem. B 112, 14772 (2008).

${ }^{28}$ L. Porcar, K. Hong, P. D. Butler, K. W. Herwig, G. S. Smith, Y. Liu, and W.-R. Chen, J. Phys. Chem. B 114, 1751 (2010).

${ }^{29}$ Y. Liu, L. Porcar, K. Hong, C.-Y. Shew, X. Li, E. Liu, P. D. Butler, K. W. Herwig, G. S. Smith, and W.-R. Chen, J. Chem. Phys. 132, 124901 (2010).

${ }^{30}$ Y. Liu, C.-Y. Chen, H.-L. Chen, K. Hong, C.-Y. Shew, X. Li, E. Liu, P. D. Butler, K. W. Herwig, G. S. Smith, L. Porcar, and W.-R. Chen, J. Phys. Chem. Lett. 1, 2020 (2010).

${ }^{31}$ K. Hong, Y. Liu, L. Porcar, D. Liu, C. Y. Gao, G. S. Smith, K. W. Herwig, S. Cai, X. Li, B. Wu, W.-R. Chen, and E. Liu (unpublished).

${ }^{32}$ R. L. Lescanec and M. Muthukumar, Macromolecules 23, 2280 (1990).

${ }^{33}$ X. Li, M. Zamponi, K. Hong, L. Porcar, C.-Y. Shew, T. Jenkins, E. Liu, G. S. Smith, K. W. Herwig, Y. Liu, and W.-R. Chen, Soft Matter 7, 618 (2011).

${ }^{34}$ T. Li, L. Porcar, Y. Liu, P. D. Butler, and W.-R. Chen, Macromolecules 41, 8916 (2008)

${ }^{35}$ In their own activities as scientific institutions, NIST and ORNL use many different materials, products, types of equipment, and services. However, NIST and ORNL do not approve, recommend, or endorse any product or proprietary material.

${ }^{36}$ J. Yang, P. Bonnesen, Y. Liu, L. Porcar, W.-R. Chen, and K. Hong (unpublished).

${ }^{37}$ S. R. Kline, J. Appl. Crystallogr. 39, 895 (2006).

${ }^{38}$ S.-H. Chen, Ann. Rev. Phys. Chem. 37, 351 (1986).

${ }^{39}$ J.-P. Hansen and I. R. McDonald, in Theory of Simple Liquids, 3rd Ed. (Elsevier, Amsterdam, 2006).

${ }^{40}$ A. D. Meltzer, D. A. Tirrell, A. A. Jones, P. T. Inglefield, D. M. Hedstrand, and D. A. Tomalia, Macromolecules 25, 4541 (1992). 